\title{
Improving the Target Position Detection in the Crossed Array Detectors Seeker by Categorizing the FOV up to the Pulses Distribution
}

\author{
A.R.Yrfanean \\ Department of \\ ElectricalEngineering \\ Maleke-Ashtar University \\ Islamic Republic of Iran
}

\author{
M.R.Mosavi \\ Department of Electrical \\ Engineering IUST \\ University Islamic \\ Republic of Iran
}

\author{
A. Mohammadi \\ Department of \\ Aerospace Engineering \\ Maleke-Ashtar \\ University Islamic \\ Republic of Iran
}

\author{
S.Y.AlchekhYasin \\ Department of Electrical \\ Engineering IUST \\ University \\ Islamic Republic of Iran
}

\begin{abstract}
Seeking in a field of view (FOV) is influenced by the existence of jammers, noise, shine background or flying perturbations. All these factors may push the target out of the FOV and cause missing the target. In all the seekers the FOV is not fully exploited which means the target can be missed before becoming out of the FOV, this results of the nonlinearity of the reticle structure. In this paper, a novel method of the target position detection a crossed four slits or crossed array detectors (CAT) seeker will be designed, simulated and evaluated. The idea of this method depends on dividing the FOV into main regions up to a certain parameter, which is the pulses number; then, each main region will be divided into sub-regions up to a second parameter which will be the pulses distribution a spin period. The errors sources will be discussed and evaluated. Other new idea will be applied which is exploiting some area of the FOV where a part of the position data is missed in the information signal by pushing the target to the region where the information signal carries the total position data.
\end{abstract}

\section{Keywords:}

Infrared Seeker, Target Position Detection, Crossed Array Detectors (CAT), Field of view (FOV).

\section{INTRODUCTION}

The infrared seeker is the system which can seeks the target by following its infrared radiation. This task is achieved by passing the infrared radiation through the reticle after focusing it by an optical system. The reticle structure reforms this continue signal into a new signal which is detected in a detector and carries in its parameters the information about the target position in the field of view (FOV). All the useful data is extracted from this information signal (IS) by a block and this block gives the correction commend to the gyro in a direction and to the autopilot in other direction [1-4].

The target position detecting block (TPB) is an important block as it derives the target position (xt,yt) in the FOV,or in the reticle plan, from IS. The precision of this process and the largeness of its work region in the FOV, which is the region where the block has the ability to derive the position correctly from the signal, are important parameters in the counter countermeasure (IRCCM) ability, and in overcoming the effect of the system perturbation on the seeker processes. The large area of the work region helps the seeker to don't miss the target when the system becomes under strong perturbations, also in the case of late flare detection where the flare takes enough time to push the target out of the work region of the FOV. In this paper, a novel method will be stated to improve the work region in crossed array detectors (CAT) seeker or in a crossed four slits reticle [5-7].

The structure of the crossed ship reticle or the crossed four slits reticle (Actually it is equivalent to a reticle with four crossed array detectors), which is a stationary reticle type, will be stated with the design parameters. This type employs a fixed reticle, with radius $\mathrm{R}_{\mathrm{a}}$ and $\mathrm{N}=4$ transparent sectors or slits, and a slightly tilted rotating mirror or lens (with spinning frequency $f_{m}$ ) to sweep the Target Image Spot (TIS) along a circular path on the reticle Target Imaging Circle (TIC) with constant radius $\left(\mathrm{R}_{\mathrm{N}}\right)$, as shown in Fig. 1. The distance of the non-concentric TIC centre and its phase relatively to the reticle centre define the position of the target in the FOV [6-10]. In Fig 1 the defined parameters:

- D: the spoke width or the detector width (pixel).

- L: the spoke length or the detector length (pixel).

- Ra: the reticle radius (FOV radius) (pixel).

- $\mathrm{R}_{\mathrm{N}}$ : the target imaging circle (TIC) (pixel).

In this study, theses parameters take the following values: $(\mathrm{D}=70, \mathrm{~L}=850, \mathrm{RN}=615, \mathrm{Ra}=885)$ unit or pixel.

The work region in the usual conscan reticles, which is called linear region in this case, is defined as the region where the information signal is pure FM modulated, this happens when the target image circle (TIC) locates totally on the reticle [8-10]. When a part of the TIC is pushed out of the reticle the signal becomes (AM/FM) modulated, so the position detecting process becomes complex. Otherwise, in the crossed array detectors (CAT) or the crossed four slits reticle the signal is not FM modulated but PPM modulated one. In this case, the region work defined as the region where the number of pulses in one spin period equals to the number of the detectors in the crossed array, which is 4 , or the number of the reticle slits in case of one detector and crossed reticle shape. As result of that, it is the region where the TIC intersects all the detectors, or the slits, as shown in Fig. 2 (a). Out of this region, at least one of the spoke of the cross cannot intersect the TIC so one or more pulses will be missed in each spin period, as shown in Fig. 2 (b). Actually, 4 
pulse positions are used to calculate the target position in the classic relations [11-16]:

$h 1=\frac{1}{2} \frac{2 \pi(d 4-d 3)}{R c}, h 2=\frac{1}{2} \frac{2 \pi(d 2-d 1)}{R c}$

$x t=R_{N} * \cos (h 1), y t=R_{N} * \cos (h 2)$

Where $\mathrm{d} 1, \mathrm{~d} 2, \mathrm{~d} 3$ and $\mathrm{d} 4$ are the pulse position in the spin period. Clearly, these relations are applicable in the 4-pulses region. Otherwise, out of the 4-pulses region these relations become inapplicable. As a result, using the classical relations the work region is the 4-pulses region.

In this task we will try to derive the target position in all the FOV, that means inner the 4-pulses region, the 3-pulses region, the 2-pulses region and the 1-pulse region.

All the modeling and simulation tasks and processes will be designed and developed using the MATLAB ${ }^{\circledR}$ tools and packages.

\section{PRINCIPLE AND RELATIONS}

For this task it is useful to define the following parameters which are clarified in Fig. 3:

- $\mathbf{T m}:$ the spin period.

- $\quad \mathbf{a}, \mathbf{b}, \mathbf{c}, \mathbf{d}$ : the 4 pulses positions relatively to the beginning instant of the spin period.

- w1, w2, w3, w4: the 4 pulses width respectively.

- $\quad \mathbf{z x 1}, \mathbf{z y 1} \mathbf{z x 2}, \mathbf{z y 2}$ : the 4 pulses width which are necessary for deriving $\mathbf{x t}$ and $\mathbf{y t}$. It is important to note that $\mathbf{z x 1}$ is not the width of the first pulse a necessarily, so on for the others. For example, in Fig. 4 (a) $\mathbf{z x 1 = w 1}$ is the width of the first pulse $\mathbf{a}$ as it is responsible to determine the target $\mathbf{x t 1 0}$ position, while in Fig. 4 (b) $\mathbf{z x 1}=\mathbf{w} 2$ is the width of the second pulse $\mathbf{b}$ as it is, in this case, responsible to determine the target $\mathbf{x t 1 0}$ position.

- fx1, fy1, fx2, fy2: the 4 pulses positions relatively to the 4 parts of the spin period. They can be negative or positive. The relations determining these parameters can be written in the general case as:

$\mathrm{fx} 1=\mathrm{F} 1(\mathrm{a}, \mathrm{b}, \mathrm{c}, \mathrm{d}, \mathrm{Tm}) \quad, \mathrm{zx} 1=\mathrm{H} 1(\mathrm{w} 1, \mathrm{w} 2, \mathrm{w} 3, \mathrm{w} 4)$

fy $1=\mathrm{F} 2(\mathrm{a}, \mathrm{b}, \mathrm{c}, \mathrm{d}, \mathrm{Tm}) \quad, \mathrm{zx} 1=\mathrm{H} 2(\mathrm{w} 1, \mathrm{w} 2, \mathrm{w} 3, \mathrm{w} 4)$

$\mathrm{fx} 2=\mathrm{F} 3(\mathrm{a}, \mathrm{b}, \mathrm{c}, \mathrm{d}, \mathrm{Tm}) \quad, \mathrm{zx} 1=\mathrm{H} 3(\mathrm{w} 1, \mathrm{w} 2, \mathrm{w} 3, \mathrm{w} 4)$

fy $2=\mathrm{F} 1(\mathrm{a}, \mathrm{b}, \mathrm{c}, \mathrm{d}, \mathrm{Tm}) \quad, \mathrm{zx} 1=\mathrm{H} 1(\mathrm{w} 1, \mathrm{w} 2, \mathrm{w} 3, \mathrm{w} 4)$

For example, in the case of Fig.4 (a) we have:

$\mathrm{fx} 1=\mathrm{a}-0.25 * \mathrm{Tm}, \quad$ fy $1=\mathrm{b}-0.5 * \mathrm{Tm}$,

$\mathrm{fx} 2=-\mathrm{c}+0.75 * \mathrm{Tm}, \quad$ fy $2=-\mathrm{d}+\mathrm{Tm}$.

$\mathrm{zx} 1=\mathrm{w} 1, \quad \mathrm{zy} 1=\mathrm{w} 2$,

$\mathrm{zx} 2=\mathrm{w} 3, \quad \mathrm{zy} 2=\mathrm{w} 4$

While inthe case of Fig.5 (b) we have:

$\mathrm{fx} 1=\mathrm{b}-0.25 * \mathrm{Tm}, \quad$ fy $1=-\mathrm{a}$,

$\mathrm{fx} 2=-\mathrm{d}+0.75 * \mathrm{Tm}, \quad$ fy $2=\mathrm{c}-0.5 * \mathrm{Tm}$.

$\mathrm{zx} 1=\mathrm{w} 2, \quad \mathrm{zy} 1=\mathrm{w} 1$,

$\mathrm{zx} 2=\mathrm{w} 4, \quad \mathrm{zy} 2=\mathrm{w} 3$

- $\phi \mathbf{x} 1, \phi \mathbf{y} 1, \phi \mathbf{x} 2, \phi \mathbf{y} 2$ : the 4 angles corresponding to the arcs $\mathbf{f x} \mathbf{1}, \mathbf{f y} \mathbf{1}, \mathbf{f x} \mathbf{2}$ and $\mathbf{f y} \mathbf{2}$ respectively. They can be negative or positive.
- Np: One of the important parameters is the number of pulses exist in a spin period; up to this parameter the FOV will be divided into a group of regions each one has its special process. As result, four main regions will be processed:

Main-Region1 (MR1): $\mathrm{Np}=1$,

Main-Region2 (MR2): $\mathrm{Np}=2$,

Main-Region3 (MR3): $\mathrm{Np}=3$,

Main-Region4 (MR4): $\mathrm{Np}=4$.

- $\quad \mathbf{q} 1, \mathbf{q} 2, \mathbf{q 3}, \mathbf{q 4}$ : these 4 secondary parameters takes the value 0 or 1 , each one of them characterizes the existence of pulses in one of the four quarters of the spin period. So $(q 1=1)$ means that there is at least one pulse in the first quarter of the spin period, while ( $\mathrm{q} 1=0)$ means that there is no pulse in this quarter. It is clear that these parameters takes their values up to the pulse positions (a, b, c, d). Actually, up to these secondary parameters each one of the four main region of the FOV will be divided in group of sub-regions. And as a result, each one will be divided into 16 sub-region, but some of these regions cannot be exist logically; for example, the case of (MR4, $\mathrm{q} 1=\mathrm{q} 2=\mathrm{q} 3=\mathrm{q} 4=0$ ) cannot be exist. For example, in the case of

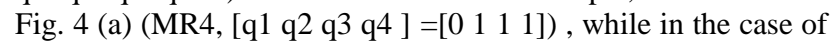

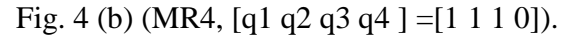

The target position (xt,yt) can be derived from the following relations:

$$
\begin{aligned}
& \mathrm{x} 10=\mathrm{RN} * \sin (\phi \mathrm{x} 1)=\mathrm{RN} * \sin \left(2 * \mathrm{pi}^{*}(\mathrm{fx} 1) / \mathrm{Tm}\right) \\
& \mathrm{x} 20=\mathrm{RN} * \sin (\phi \mathrm{x} 2)=\mathrm{RN} * \sin \left(2 * \mathrm{pi}^{*}(\mathrm{fx} 2) / \mathrm{Tm}\right) \\
& \mathrm{y} 10=\mathrm{RN} * \sin (\phi \mathrm{y} 1)=\mathrm{RN}^{*} \sin \left(2 * \mathrm{pi}^{*}(\mathrm{fy} 1) / \mathrm{Tm}\right) \\
& \mathrm{y} 20=\mathrm{RN} * \sin (\phi \mathrm{y} 2)=\mathrm{RN}^{*} \sin \left(2 * \mathrm{pi}^{*}(\mathrm{fy} 2) / \mathrm{Tm}\right) \\
& \text { In general, it can be written that: } \\
& \mathrm{xt}=\mathrm{G} 1(\mathrm{x} 10, \mathrm{x} 20) \\
& \mathrm{yt}=\mathrm{G} 2(\mathrm{y} 10, \mathrm{y} 20)
\end{aligned}
$$

Finally, each point from the FOV can be characterized by 8 main parameters $(a, b, c, d, w 1, w 2, w 3, w 4)$ from whom the secondary parameters can be derived $(\mathrm{Np}, \mathrm{q} 1, \mathrm{q} 2, \mathrm{q} 3, \mathrm{q} 4, \mathrm{fx} 1$, $\mathrm{fx} 2, \mathrm{fy} 1, \mathrm{fy} 2, \mathrm{zx} 1, \mathrm{zx} 2, \mathrm{zx} 3, \mathrm{zx} 4)$. In addition to that, the FOV can be divided into 4 main regions and each one can be divided to a group of sub-regions up to the distribution of the pulses among the quarters of the spin period. For this purpose a model will be prepared which gives these parameters for all the node points of an appropriate mesh of the FOV and store them in a file to get them easily during testing the method of target position detection.

A Simulink model is prepared to get the main and secondary parameters of the signal resulting for each node point in an appropriate mesh on the FOV. This model uses the CAT reticle model which takes the permanent reticle design parameters and the target position in addition to the spot radius Tr. This model derives the main parameters for all the points of the mesh on the FOV, then all the data are stored in an excel file. This data file is special for one spot radius Tr. So two files are achieved, one for the minimum radius value $(\mathrm{Tr}=1)$ and the other is for the maximum case $(\mathrm{Tr}=35)$.

The task will achieved by dividing the FOV into good category and then finding the relations for extraction the exist data in the information signal in each sub-region in the FOV. 


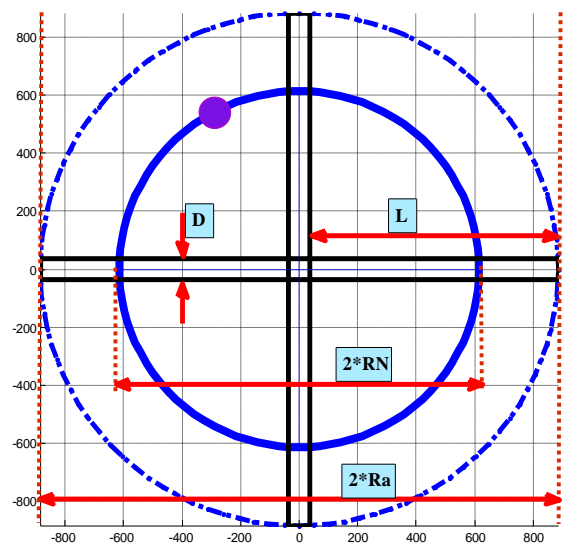

(a)

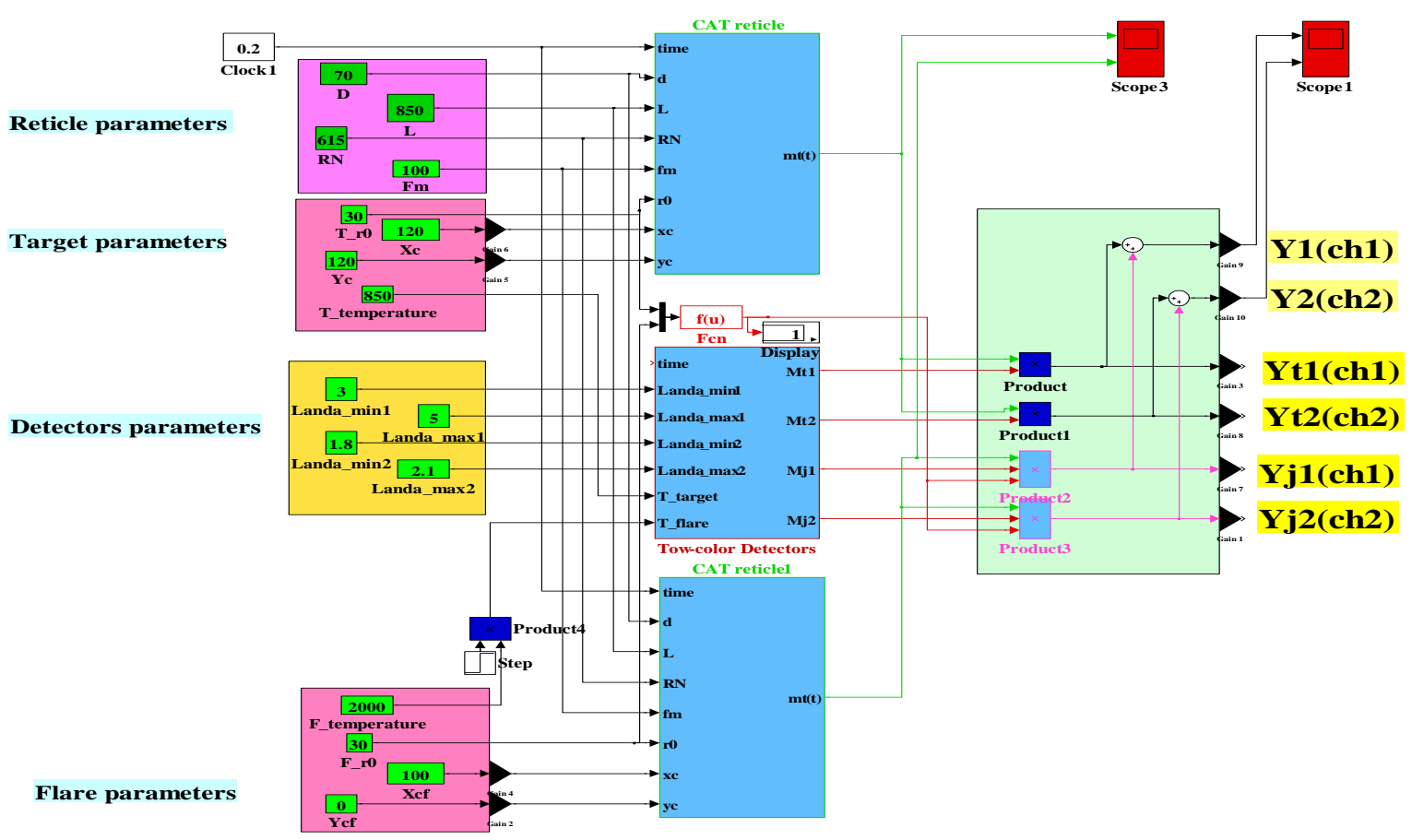

(b)

Fig. 1: (a) the design parameters of a CAT reticle (D,L, Ra, RN). (b) the seeker model with the inputs (reticle parameters, detectors parameters, target parameters, and flare parameters)and the outputs ( Y1(ch1), Y2(ch2), Ref=Y1T, Y2T, Y1F, Y2F ).
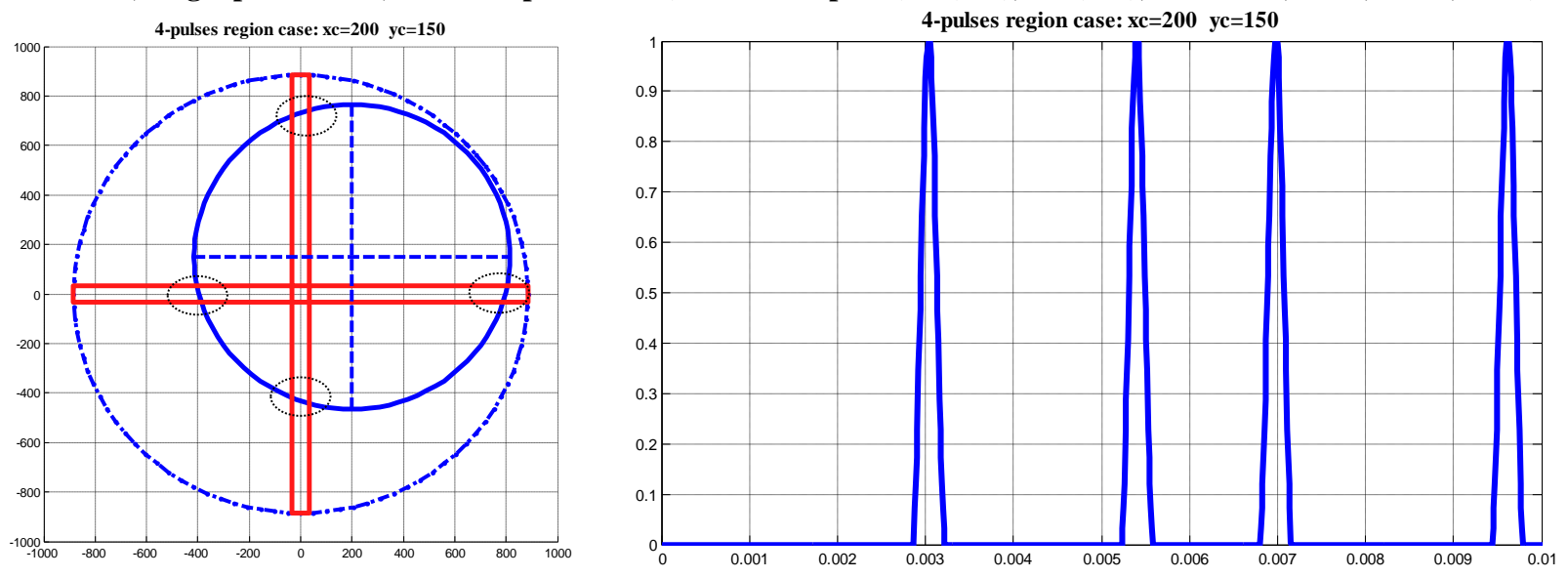
(a) 4-pulses region case ( $\mathrm{xc}=200$ pixels, $\mathrm{yc}=150$ pixels).
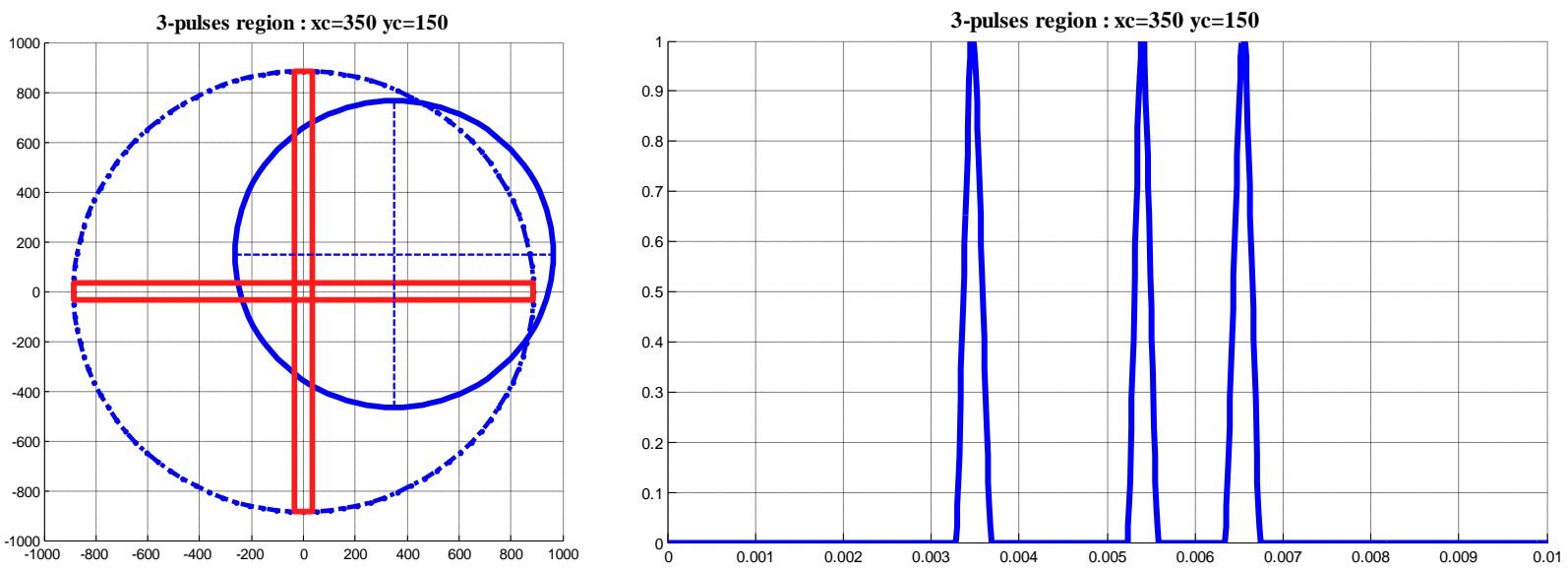

(b) 3-pulses region case ( $x c=350$ pixels, $y c=150$ pixels).

Fig. 2 The field of view regions up to the pulses number in one spinning period.

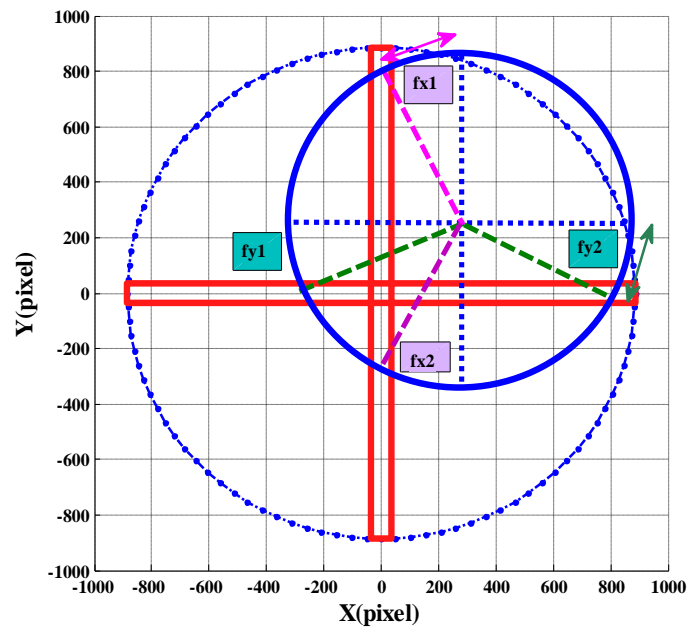

(a)

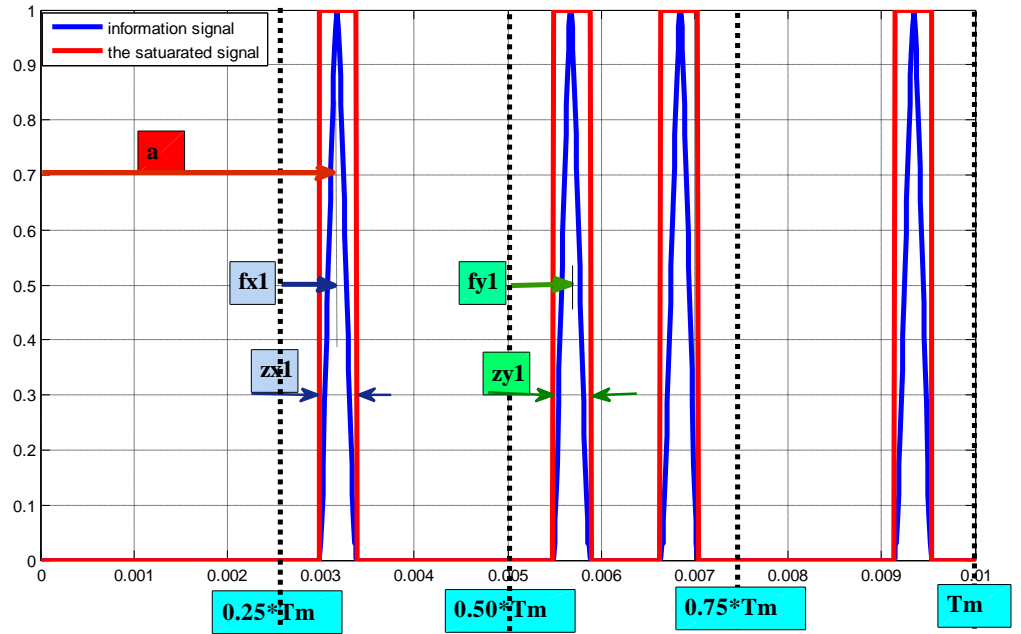

(b)

Fig. 3: The parameters defining the target position detection (a, b, c, d, fx1, fx2, fy1, fy2, zx1, zx2, zy1, zy2,Tm).

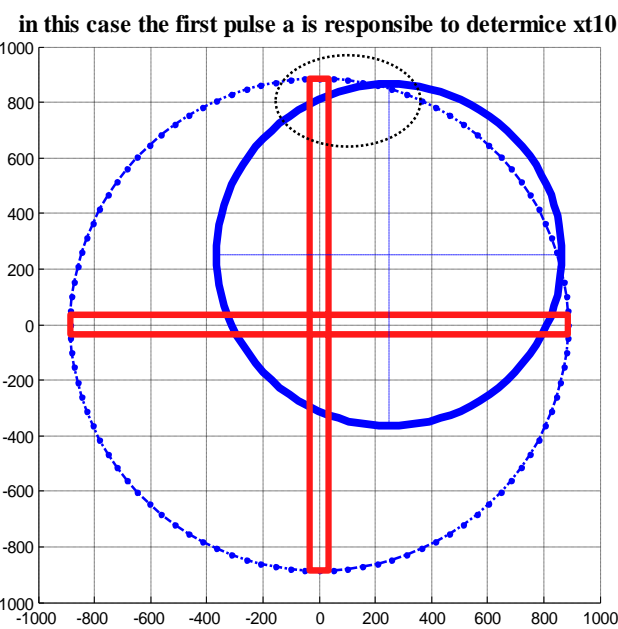

(a)

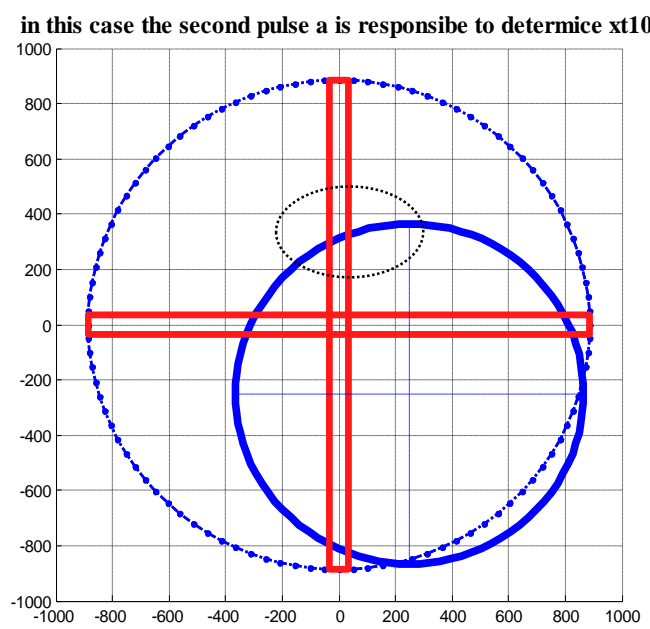

(b) 
Fig. 4: The responsible pulse of determining the target position xt10: (a) the first pulse is the wanted pulse (b) the second pulse is the wanted pulse.

\section{THE MAIN REGIONS IN THE FOV}

Two cases are stated for these regions up to the thermal spot radius, the maximum $(\mathrm{Tr}=35$ pixel) and the minimum $(\mathrm{Tr}=1$ pixel) values of $\mathrm{Tr}$ are considered and the results are drawn in Fig 5. It is clear that the regions depend on the thermal spot radius but the distribution is same. MR4 is in the centre FOV consists of a disc with a radius $\left(\mathrm{R}_{\mathrm{a}}-\mathrm{R}_{\mathrm{N}}\right)$ and additional thin related to the fact that the thermal sport is not an ideal point. Surrounding MR4 the other regions locate sequentially. The region, as we will see later, where the target position data is exist in the information signal is $\mathrm{M} 1=\mathrm{MR} 4 \cup \mathrm{MR} 3$. The region where a part at least is exist is M2=MR4 $\cup M R 3 \cup M R 4 \cup M R 3$. While the classic effective work region is M0=MR4.

So from Fig 5, these ratios are defined which express the exploitation of the FOV:

$$
\begin{aligned}
R 0 & =\frac{M 0}{\pi(R a)^{2}} \approx \frac{\pi(R a-R N)^{2}}{\pi(R a)^{2}} \approx 9 \% \\
R 1 & =\frac{M 0}{M 1} \approx \frac{\pi(R a-R N)^{2}}{\pi(R N)^{2}} \approx 20 \% \\
R 2 & =\frac{M 0}{M 2} \approx \frac{\pi(R a-R N)^{2}}{\pi(R a-R N)^{2}+4 * \pi(R N)^{2}} \cong \frac{R 1}{4+R 1} \approx 5 \%
\end{aligned}
$$

\section{POSITION DETECTION IN MR4}

\subsection{The Sub-Regions in the MR4}

It is clear from Fig 5 that this region is in its biggest and more complex case when the spot radius is minimum, so it is good practice to solve the problem in this case then generalized it on all the cases of spot radius. Actually, this main region is formed from three groups:

- The first group: this group of points where the TIC intersects the four slits of the reticle, as shown in Fig 6 (a).

- The second group: results from passing the TIC in the middle region of the reticle; clearly, this group appears when the spot radius is too small to stile able to intersect

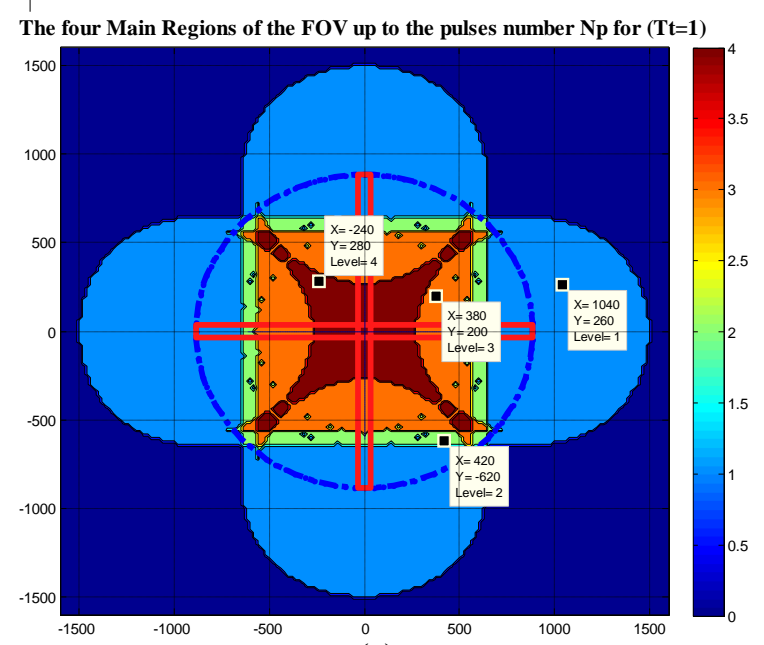

(a)

Fig. 5: The four main regions in the cases: (a) $\operatorname{Tr}=1$ pixel (b) $\operatorname{Tr}=35$ pixel.

the spokes separately in the middle region of the reticle, as show in Fig 6(b).

- The third group: this additional kind group results from intersecting the TIC with two slits each one twice, as show in Fig 6(c).

To determine the sub-regions of MR4 a m-file program is used which distinguishes 16 sub-regions up to the values of [q1 q2 q3 q4], but only 4 of them can be exist logically, these sub-regions are MR45, MR46, MR47 and MR48 as shown in Fig 7(a).

As a result for these 12 cases, the whole problem becomes finding the functions $\{\mathrm{F} 1, \mathrm{~F} 2, \mathrm{~F} 3, \mathrm{~F} 4, \mathrm{H} 1, \mathrm{H} 2, \mathrm{H} 3, \mathrm{H} 4, \mathrm{G} 1, \mathrm{G} 2\}$ of the relations (4-7) and the relations (12-13) in each case. The solution of MR45 is stated and shown in Fig 7, and the others will be in the same manner.

\subsection{The Target Position Error in the MR4}

For evaluating this method in the MR4, all target positions in all the point of MR4 is gotten using these relations above, then this data is compared with the exact positions. Mostly the region the data is exact, but at the limitation of it error appear.

The first one results when the pulse width become large and the middle of the pulse in the time domain cannot expresses the position of the centre of the interaction of the slit. This error is too small to take into account, especially that it appears when $\mathrm{xc}$ or yc takes big values $(\mathbf{x c}>\mathbf{1 5 0})$.

Actually, this error is corrected by deriving the xc corresponding the beginning and the ending point of the pulse and then take the average.

The second error happens in case of all the points where the TIC intersects one of the outer limits of the spokes reticle. That means that the pulse in formed partially and the pulse position and width could not be measured correctly. This is solved by switching between $\mathrm{x} 10$ and $\mathrm{x} 20$.

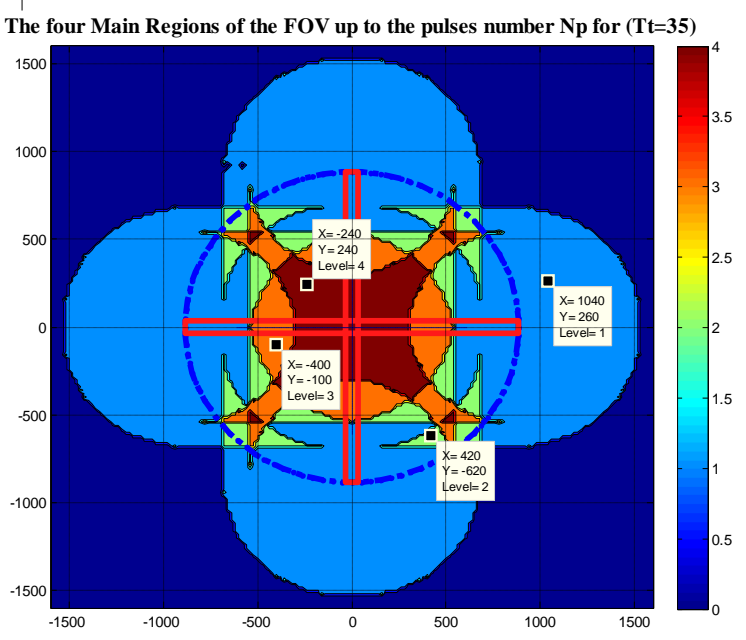

(b) 


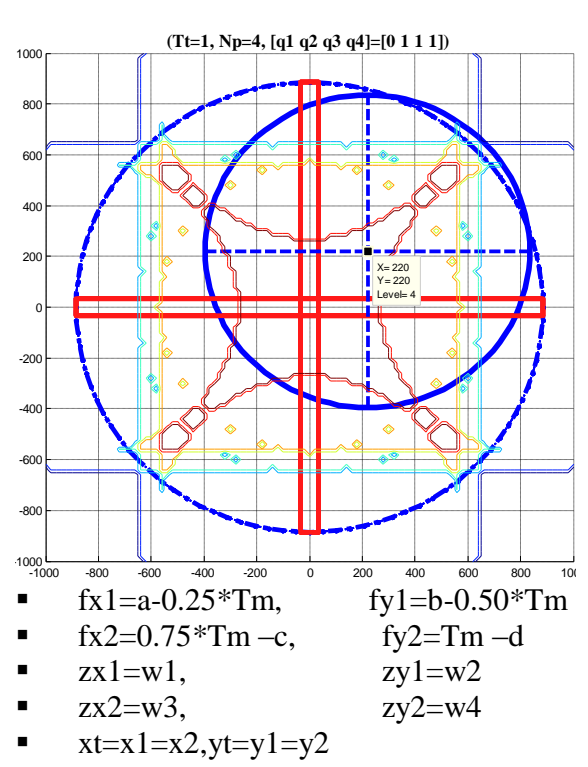

(a)

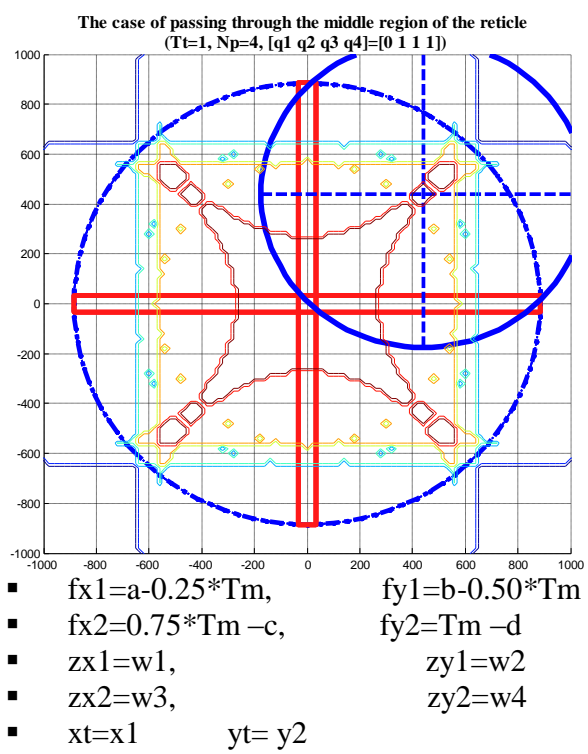

(b)

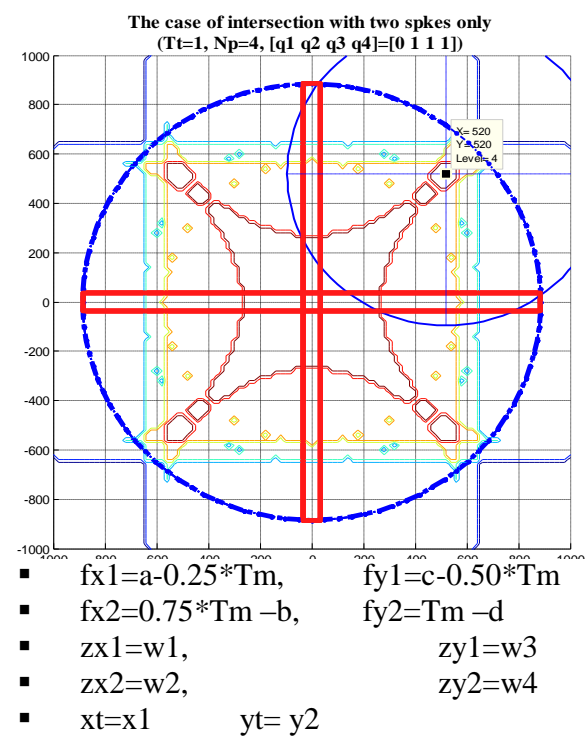

(c)

Fig. 6 the three kinds of groups in the 4-pulses main region: (a) TIC intersect with all the spokes, (b) TIC intersect with all the spokes with passing in the middle region of the reticle, (c) TIC intersects with two spokes each one twice.

The important note is that, this error appears on $\mathrm{x}$ when $\mathrm{y}$ takes largest values in MR4 and vice versa, so the phase doesn't influence.Clearly. $(\mathrm{dx}<1$ and $d y<1)$ in most of MR4. But in thin limitation area of MR4 $(\mathrm{dx}<25, \mathrm{dy}<25)$; taking into account that in this area $(x>250, y>250)$ and the area is so thin the result is ultra-accepted.

\section{POSITION DETECTION IN MR3}

\subsection{The Sub-Regions in the MR3}

It is clear from Fig 5 that this region is in its biggest and more complex case when the spot radius is maximum, so it is good practice to solve the problem in this case then generalized it on all the cases of spot radius. Actually, this main region is formed from three groups:

- The first group: in this group the TIC intersects the three slits of the reticle, as shown in Fig 8 (a).

- The second group: results from passing the TIC in the middle region of the; clearly, this group appears when the spot radius is large to be able to not intersect the spokes separately in the middle region of the reticle, as show in Fig 8(b).

- The third group: this additional kind group results by intersecting the TIC of two spokes one of them twice, as show in Fig 8(c). 
Sub regions of the 4-pulses region up to the secondary parameters $(\operatorname{Tr}=1)$

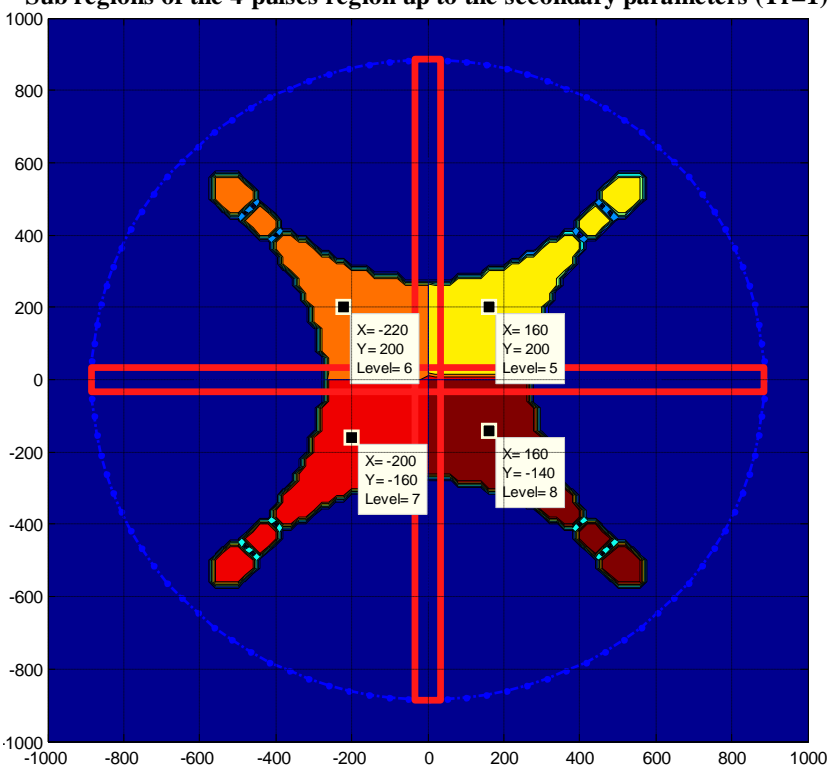

(a)

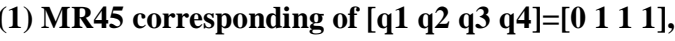

(2) MR46 corresponding of [q1 qq2 q3 q4] $=\left[\begin{array}{llll}1 & 0 & 1 & 1\end{array}\right]$,

(3) MR47 corresponding of [q1 q2 q3 q4 $]=\left[\begin{array}{llll}1 & 1 & 0 & 1\end{array}\right]$,

(4) MR48 corresponding of [q1 q2 q3 q4 $]=\left[\begin{array}{llll}1 & 1 & 1 & 0\end{array}\right]$.

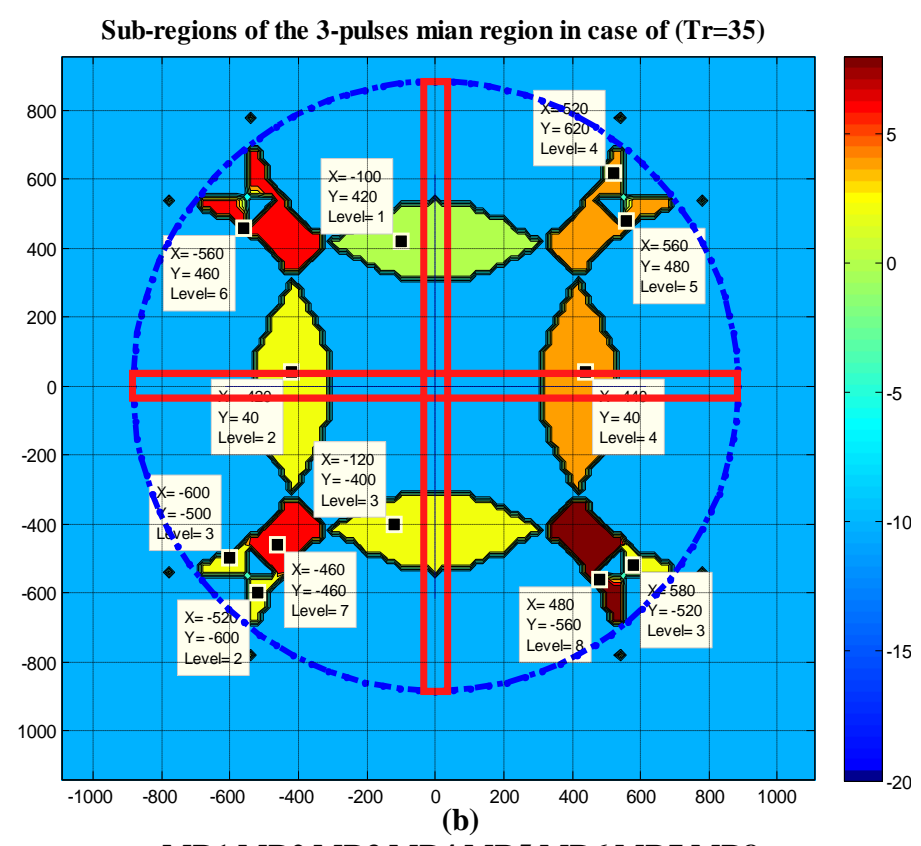

MR1,MR2,MR3,MR4,MR5,MR6,MR7,MR8

Fig. 7: (a) the four sub regions of the 4-pulses main region, (b) the sub regions of the 3-pulses main region,

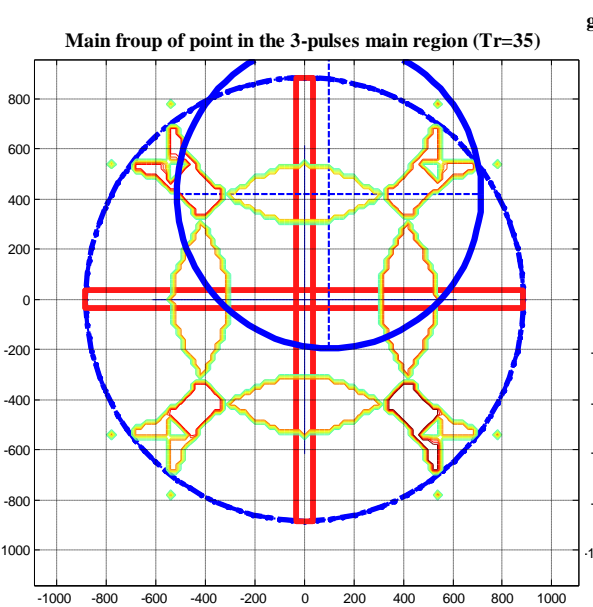

(a)

- $\mathrm{fx} 1=0.75 * \mathrm{Tm}-\mathrm{b}$

- $\mathrm{fx} 2=$ none,

- $\mathrm{zx} 1=\mathrm{w} 2$,

- $\mathrm{zx} 2=$ none,

- $\mathrm{xt}=\mathrm{x} 1$,

fy $1=\mathrm{a}-0.50 * \mathrm{Tm}$ fy $2=\mathrm{Tm}-\mathrm{c}$ $\mathrm{zy} 1=\mathrm{w} 1$ $\mathrm{zy} 2=\mathrm{w} 3$ $\mathrm{yt}=\mathrm{y} 1=\mathrm{y} 2$,

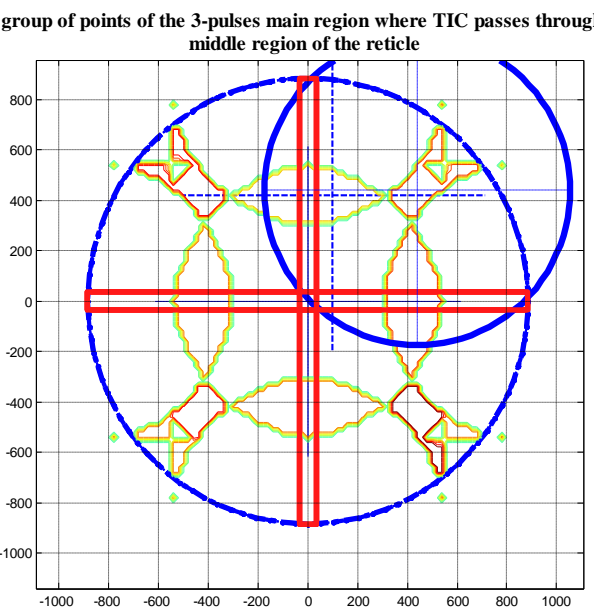

(b)

- $\mathrm{fx} 1=\mathrm{a}-0.25 * \mathrm{Tm}$

fy $1=b-0.50 * \mathrm{Tm}$
- $\mathrm{fx} 2=-\mathrm{b}+0.75 * \mathrm{Tm}$

- $\quad \mathrm{zx} 1=\mathrm{w} 1$,

- $\quad \mathrm{zx} 2=\mathrm{w} 2$,

- $\mathrm{xt}=\mathrm{x} 1$, fy $2=\mathrm{Tm}-\mathrm{c}$

$\mathrm{zy} 1=\mathrm{w} 2$

$\mathrm{zy} 2=\mathrm{w} 3$

$\mathrm{yt}=\mathrm{y} 2$,

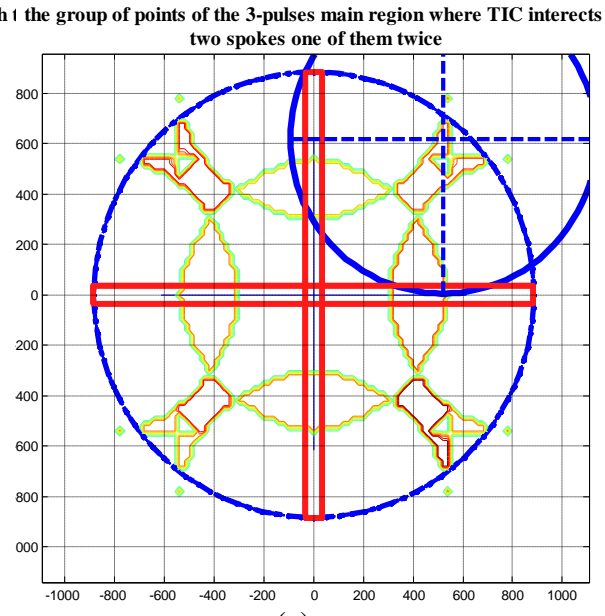

(c)

if (a>0.47*Tm)

fx $1=-0.25 * \mathrm{Tm}+\mathrm{a} ; \quad$ fy $1=-0.50 * \mathrm{Tm}+\mathrm{b}$

- $\mathrm{fx} 2=\mathrm{f} \times 1$

- $\mathrm{zx} 1=\mathrm{w} 1$,

- $\quad \mathrm{zx} 2=\mathrm{w} 1$

- $\mathrm{xt}=\mathrm{x} 1$, fy $2=\mathrm{Tm}-\mathrm{c}$

$\mathrm{zy} 1=\mathrm{w} 2$;

$\mathrm{zy} 2=\mathrm{w} 3$;

$\mathrm{yt}=\mathrm{y} 2$

Fig. 8 The three kinds of groups in the 3-pulses main region: (a) TIC intersects with three spokes, (b) TIC intersect passes in the middle region of the reticle,

(c) TIC intersects with two spokes one them twice. 


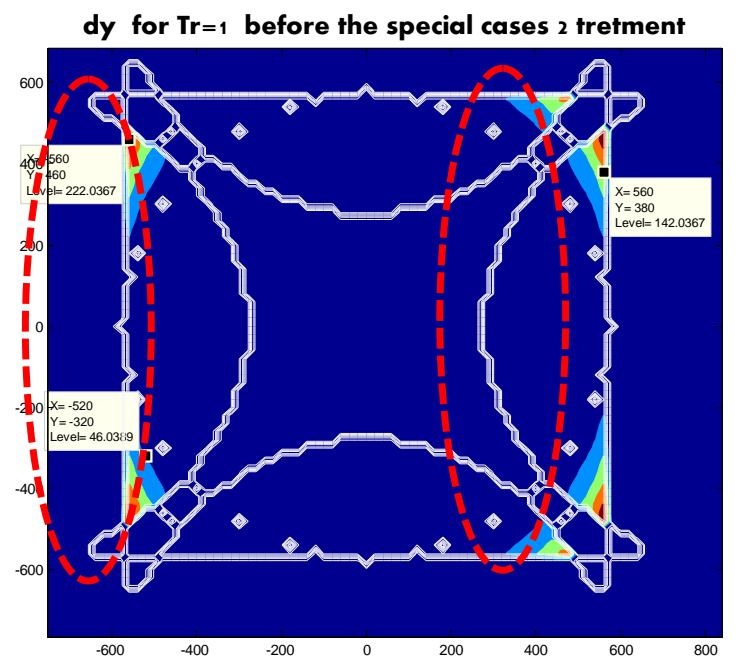

(a)

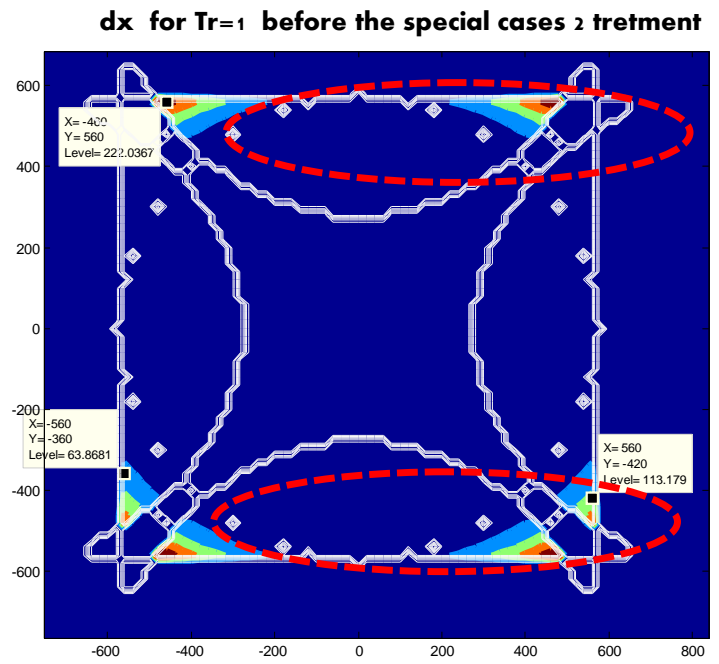

(b)

Fig. 9 The special cases for small spot radius: (a) the region where large yt error (b) the region where large yt error.

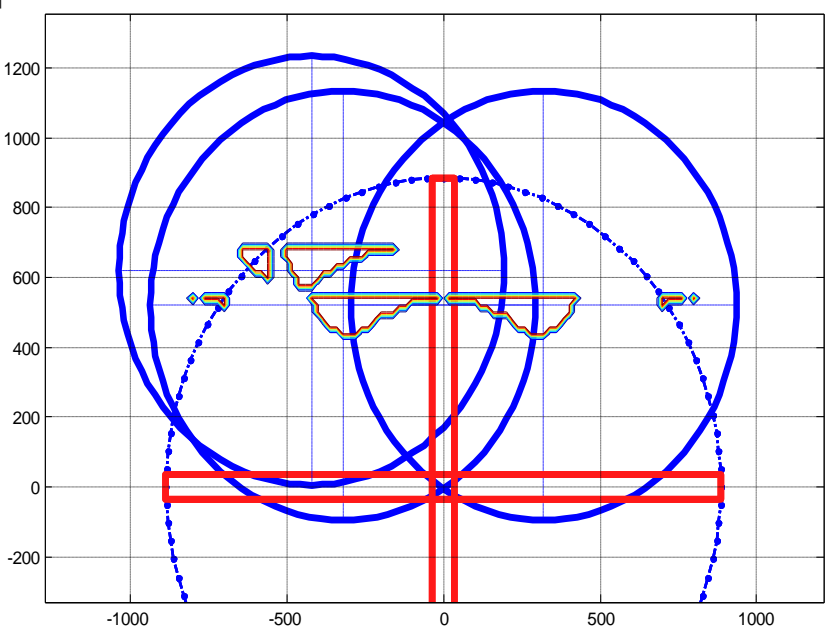

Fig 10: Cases of MR2 where the data needed to find xt is missed.

To determine the sub-regions of MR3 a m-file program is used which distinguishes 16 sub-regions up to the values of [q1 q2 q3 q4], but only 8 of them can be exist logically, these sub-regions are MR31, MR32, MR33, MR34, MR35, MR36, MR37 and MR38 as shown in Fig 7(b). The solution of MR31 is stated and shown in Fig 8, and the others will be in the same manner.

\subsection{The Target Position Error in the MR3}

Actually, after modulating and simulating all the previous relation for MR3 and applying it for small spot radius $(\operatorname{Tr}<20$ pixel) a group of points give unacceptable results and large error, as show in Fig 9. This figure shows that a certain region of large error in determining xt appears in the main area. Similarly, a certain region for large error in yt determining appears in different place. This results from the confusion between certain areas. Adding certain conditions is made to solve this confusion.Clearly. $(\mathrm{dx}<1$ and $\mathrm{dy}<1)$ in most of MR3. But in thin limitation area of MR3 $(\mathrm{dx}<65, \mathrm{dy}<65)$; taking into

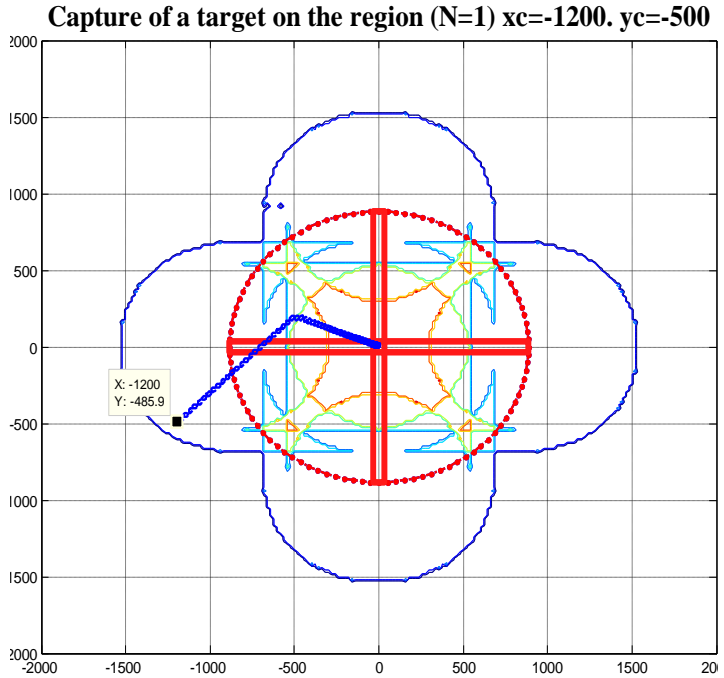

Fig 11: Combining guidance by direction and position.

account that in this area $(x>550, y>550)$ and the area is so thin the result is ultra-accepted as only one of $\mathrm{xt}$ or yt has error in the same position.

\section{POSITION DETECTION IN MR2 AND MR1}

Actually, in MR4 and MR3 the target position is extracted exactly which means the two parts (xt, yt) or (rt, $\varphi t)$. In this region $M 1=M R 4 \cup M R 3$ the information signal contains the target position detection in MR2 and MR1.

On other hand in MR2 or MR1 one of the two data is missed $\mathrm{xt}$ or yt. This results from the fact that the TIC misses the interaction of the two slits responsible of extracting this part of data. Fig 10 shows cases of MR2 where the data of finding $x t$ is missed and only yt can be gotten. For solving this problem, a new method is applied that is the guidance by direction instead of guidance by position. The idea is that it is enough to find the 
direction to push the target from MR2 or MR1 to arrive M1, and after arriving M1 the guidance by position will continue the task. For example, for the case of Fig 11:

$\mathrm{f} \times 1=0$;

fy $2=1000-b ; \quad z y 2=z 2 ;$

fy $1=a-500 ; \quad z y 1=z 1$;

Fig 15 shows how the target start from the $(\mathrm{xc}=1200$, $\mathrm{yc}=500)$ and after start seeking the target follows a suitable direction to arrive M1, after that exact target position continues in the task. As a result the new values of exploitation of FOV:

NewR $0=\frac{\pi(R a)^{2}}{\pi(R a)^{2}}=100$
NewR $1=\frac{M 1}{M 1} 100 \%$
NewR2 $=\frac{M 2}{M 2} 100 \%$

\section{CONCLUSION}

Improving the target position in the FOV and extension of the effective work region is important task that took efforts in several works either by estimation or by generalized relations. In this paper, the work is on the CAT seeker. Firstly, the work region which was a part of MR4 is improved to cover all MR4 by finding new relations. Then, the MR3 is divided into suitable category which helps to discuss each case separately. By adding the MR3 to the effective work region, all the region of the FOV where the information signal carries the position data is fully exploited. For the rest of the FOV where a part of the position data is missed a new technique is used by choosing a suitable direction to push the target back to the region where the position data is available. By combining these two methods in the two different regions, the position and the direction regions of the FOV, all the FOV becomes available. Finally, errors sources in discussed and the relations are modulated; then the position errors are evaluated by comparing the exact position with measured one.

\section{REFERENCES}

[1] G. Gerson and A. K. Rue, "Tracking Systems," Chap. 22 in The Infrared Handbook, G. J. Zissis and W. L. Wolfe, Eds., RIM, Ann Arbor, MI(1985).

[2] K. Seyrafi and S. A. Hovanessian, Chap. 7 in Introduction to Electooptical Imaging and Tracking Systems, pp. 193220, ArtechHouse,Norwood, MA(1993).

[3] R. Legault, "Reticle and Image Analyses," Chap. 17 in The Infrared Handbook, revised ed., W. L. Wolfe and G. J. Zissis, Eds., pp. 17-1-17-49, Environmental Research Institute of Michigan, Ann Arbor, MI(1985).

[4] J. S. Accetta and D. L. Shymaker, Eds., "The Infrared \& Electro-Optical Systems Handbook”, SPIE Press (1993).
[5] J. S. Oh, K. S. Doo, S. G. Jahng, and J. S. Choi, "A New Counter-Countermeasure Algorithm for Two-Color Infrared Seekers," Optical Engineering, vol. 40, No. 8 (2001).

[6] R. G. Driggers, C. E. Halford, G. D. Boreman, D. Lattman, and K. F. Williams, "Parameters of Spinning FM Reticles," Appi. Opt. 30, 887-895(1991).

[7] P. E. Mengers and K. B. O'Brien, "Analysis of Error Response of Amplitude Modulated Reticles," J. Opt. Soc. Am. 54, 668-671 (1964).

[8] Z. W. Chao and J. L. Chu, "General Analysis of FrequencyModulation Reticles," Opt. Eng. 27, 440-442 (1988).

[9] M. A. Porras, J. Alda, and E. Bernabeu, "AmplitudeModulated and Frequency-Modulated Reticle Responses of Gaussian Beams," Optical Engineering, Vol. 30, No. 12 (1991).

[10] R. G. Driggers, C. E. Halford, and G. D. Boreman, "Use of Spatial Light Modulators in Frequency Modulation Reticle Trackers," Optical Engineering. Vol. 29, No. 11, pp. 13981403 (1990).

[11] W. Haifeng, L. Zhi, Z, Qing, and S. Xinzhi, "A Double Infrared Image Processing System Using Rosette Scanning," Proceeding. SPIE, Vol. 2894, pp. 2-10 (1996).

[12] S. G. Jahng, H. K. Hong, S. H. Han, and J. S. Choi, "Dynamic Simulation of The Rosette Scanning Infrared Seeker and an IRCCM using The Moment Technique," Opt. Eng. 38(5), 921-928 (1999).

[13] J. S. Oh, K. S. Doo, S. G. Jahng, D. S. Seo, and J. S. Choi, "Novel Adaptive Digital Signal Processing Algorithm for a Stationary Reticle Seeker," Optical Engineering, Vol. 39, No. 1 0, pp. 2797-2803, 2000.

[14] D. P. Forrai and J. J. Maier, "Generic Models in the Advanced IRCM Assessment Model," Proceeding of the 2001 Winter Simulation Conference, pp.789-796 (2001).

[15] M. R. Mosavi, M. Asadpour, and R. Kalili,"Comparing Performance of Two Infrared Anti-Jamming Methods using Fuzzy System and Neural Network," 2007 Congress on Intelligent and Fuzzy Systems, Ferdowsi University of Mashhad, Iran (2007).

[16] M. R. Mosavi, M. Asadpour, and H. A. Amerim,"Design and Simulation of an Infrared Jammer Source for an Infrared Seeker," IEEE Conference on Signal Processing, Communications, and Networking, India (2008). 